\title{
Daunorubicin and gambogic acid coloaded cysteamine-CdTe quantum dots minimizing the multidrug resistance of lymphoma in vitro and in vivo
}

\author{
This article was published in the following Dove Press journal: \\ International Journal of Nanomedicine \\ 18 October 2016 \\ Number of times this article has been viewed
}

\author{
Yi Zhou',2 \\ Ruju Wang' \\ Bing Chen' \\ Dan Sun ${ }^{3}$ \\ Yong $\mathrm{Hu}^{2}$ \\ Peipei $\mathrm{Xu}$ \\ 'Department of Hematology, Drum \\ Tower Hospital, School of Medicine, \\ ${ }^{2}$ Institute of Materials Engineering \\ and Collaborative Innovation Center \\ of Chemistry for Life Sciences, \\ College of Engineering and Applied \\ Sciences, Nanjing University, Nanjing \\ People's Republic of China; ${ }^{3}$ School \\ of Mechanical Engineering, Queen's \\ University Belfast, Belfast, UK
}

\begin{abstract}
To minimize the side effects and the multidrug resistance (MDR) arising from daunorubicin (DNR) treatment of malignant lymphoma, a chemotherapy formulation of cysteamine-modified cadmium tellurium (Cys-CdTe) quantum dots coloaded with DNR and gambogic acid (GA) nanoparticles (DNR-GA-Cys-CdTe NPs) was developed. The physical property, drug-loading efficiency and drug release behavior of these DNR-GA-Cys-CdTe NPs were evaluated, and their cytotoxicity was explored by 3-[4,5-dimethylthiazol-2-y1]-2,5diphenyltetrazolium bromide assay. These DNR-GA-Cys-CdTe NPs possessed a $\mathrm{pH}$-responsive behavior, and displayed a dose-dependent antiproliferative activity on multidrug-resistant lymphoma Raji/DNR cells. The accumulation of DNR inside the cells, revealed by flow cytometry assay, and the down-regulated expression of P-glycoprotein inside the Raji/DNR cells measured by Western blotting assay indicated that these DNR-GA-Cys-CdTe NPs could minimize the MDR of Raji/DNR cells. This multidrug delivery system would be a promising strategy for minimizing MDR against the lymphoma.
\end{abstract}

Keywords: daunorubicin, gambogic acid, Cys-CdTe QDs, multidrug resistance, non-Hodgkin's lymphoma

\section{Introduction}

Malignant lymphoma is a group of primary malignant tumor in the lymph tissues, which can be divided into Hodgkin's lymphoma (HL, 10\%) and non-Hodgkin's lymphoma (NHL, 90\%) according to the clinical and pathological features. ${ }^{1}$ In the US, it was estimated that 20,940 people died from lymphoma (HL 1,150; NHL 19,790) in 2015, and the mortality rate of NHL was ranked ninth in males and eighth in females for cancer-related deaths. ${ }^{2}$ Although, benefiting from the hematopoietic stem cell transplantation, treatment of refractory lymphoma greatly improved over the past few decades, ${ }^{3}$ chemotherapeutics are still the most effective treatment for malignant lymphoma.

Daunorubicin (DNR), an anthracycline antibiotic, has been used as a first-line chemotherapy component in the treatment of NHL. ${ }^{4,5}$ Despite its side effects, including cardiac toxicity and bone marrow suppression, ${ }^{6}$ the modest lipophicity and net positive charge at physiological $\mathrm{pH}$ made DNR an efficient substrate for P-glycoprotein (P-gp). ${ }^{7,8} \mathrm{P}$-gp is known to actively pump substrates out of tumor cells through an adenosine triphosphate, resulting in multidrug resistance (MDR) and hampering the 
successful clinic application of DNR. ${ }^{9,10}$ To date, reducing the side effects of DNR and minimizing MDR still remain major challenges in the application of DNR. ${ }^{11}$

To minimize MDR in the treatment of tumor, innovative strategies, including novel delivery systems and effective sensitizers, were applied to selectively deliver the chemotherapeutic drugs inside the tumor cells or improve the sensitivity of chemotherapy to strengthen the therapeutic efficacy. ${ }^{12}$ Among them, nanocarriers showed promising potential in minimizing P-gp-mediated MDR by extending the circulation time of drugs in vivo, delivering large amount of drugs to the targeting tissue, and releasing a high concentration of drugs inside the cells after they translocate on the cell membrane. ${ }^{13-15}$ In our previous report, gambogic acid (GA)-loaded cysteamine-modified cadmium tellurium quantum dots (GA-Cys-CdTe QDs) showed promising ability to improve drug accumulation in K562 cells and minimize MDR of the drug-resistant K562/A02 cells. ${ }^{16}$ GA not only shows significant anticancer activity in vitro and in vivo, but also is an attractive agent for the chemosensitization of cancer cells. Recent research shows that GA can decrease P-gp expression as well as reduce its activity, suggesting the potential of GA as a novel P-gp inhibitor. ${ }^{17}$ Codelivery of GA and docetaxel can restore the docetaxel sensitivity in gastric cancer cell lines and minimize the apoptotic defect of BGC-823/Doc cells. ${ }^{18}$ In addition, the codelivery of GA and DNR by polymeric nanoparticles could greatly increase the intracellular concentration of DNR and decrease the expression of $\mathrm{P}$-gp, thus offering a promising solution to minimize MDR and promote anticancer efficiency.

In this work, a multifunctional drug delivery system combined with GA, DNR, and Cys-CdTe QDs nanoparticles (DNR-GA-Cys-CdTe NPs) was fabricated. CdTe QDs have unique optical properties and have been widely used in simultaneous molecular/tissue imaging, cell labelling, in vivo tumor detection, and drug delivery applications. ${ }^{19-21}$ With the aid of surface modification with cysteamine, GA and DNR could be facilely loaded onto the CdTe QDs through electronic interaction, resulting in high drug loading. Furthermore, GA can effectively bind to transferrin receptor - a type of protein overexpressed on the surface of different cancer cells, providing GA an additional ability to target cancer cells. ${ }^{22}$ We hope these multifunctional DNR-GA-Cys-CdTe NPs can effectively deliver the drug, selectively bind to lymphoma cells, minimize MDR and monitor their intracellular distribution in real time (Figure 1).

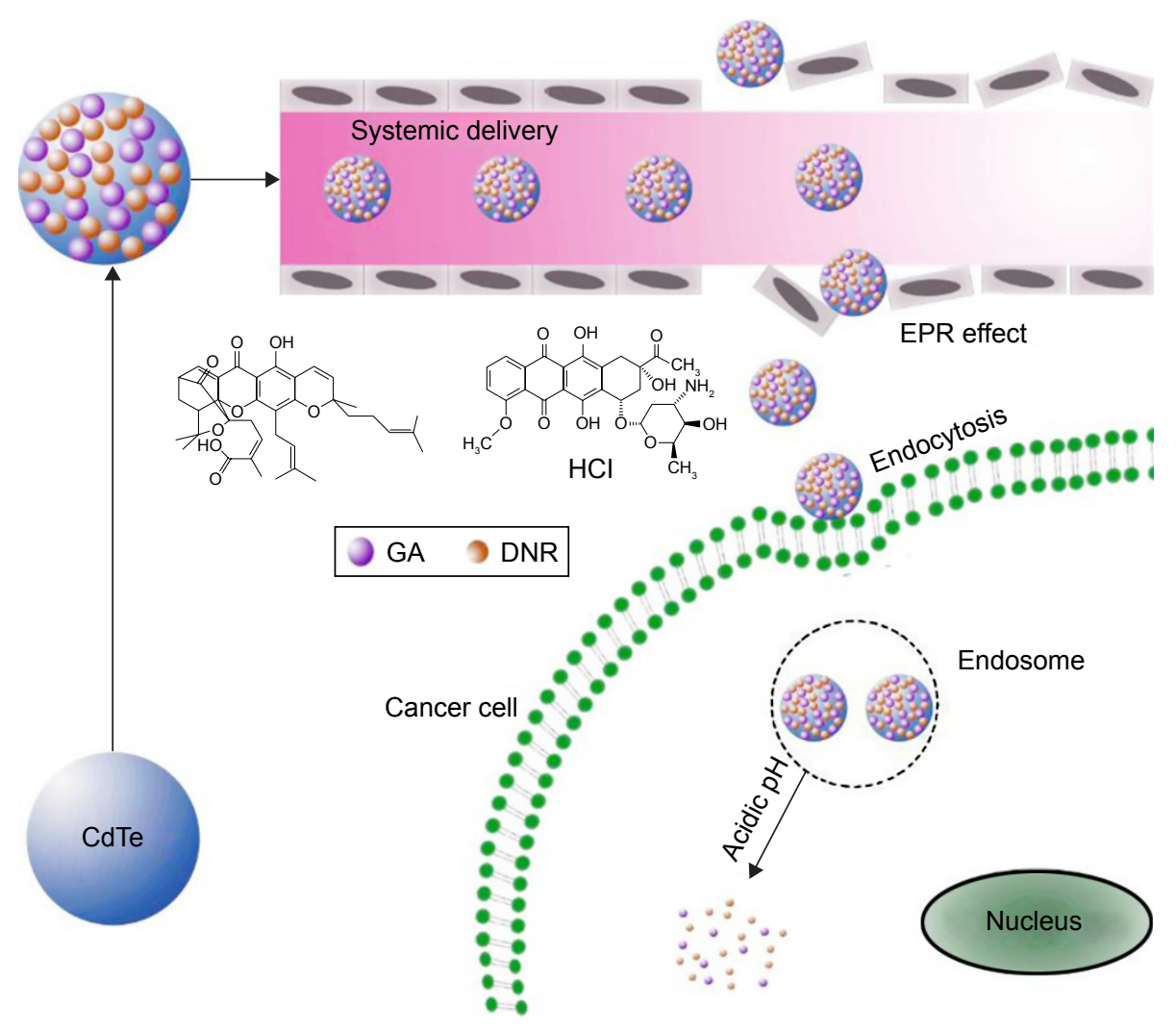

Figure I Schematic illustration of the structure of DNR-GA-Cys-CdTe NPs.

Notes: These DNR-GA-Cys-CdTe NPs can enter the cancer cells by endocytosis. Drugs can be released from DNR-GA-Cys-CdTe NPs in the acidic environment of endosomes/lysosomes to exert the synergistic antitumor effect.

Abbreviations: Cys, cysteamine; CdTe, cadmium-tellurium; DNR, daunorubicin; EPR, enhanced permeability and retention; GA, gambogic acid; NPs, nanoparticles. 
Their ability to enhance the antitumor effect of DNR and minimize MDR in lymphoma cells Raji and drug-resistant cell line Raji/DNR were studied.

\section{Materials and methods Reagents and animals}

GA (Kanion Pharmaceutical Co., Ltd, Lianyungang, People's Republic of China) was dissolved in dimethyl sulfoxide (Sigma-Aldrich Co., St Louis, MO, USA), and stored at $-20^{\circ} \mathrm{C}$. Daunorubicin hydrochloride (DNR $\left.\cdot \mathrm{HCl}\right)$ (Hisun Pharmaceutical Co., Ltd, Taizhou, People's Republic of China) was stored at $4^{\circ} \mathrm{C}$. Cys-CdTe QDs were prepared as described elsewhere, ${ }^{23}$ and was ultrasonicated for 5 minutes before use. Roswell Park Memorial Institute (RPMI) 1640 medium was purchased from Gibco Chemical Co. (Carlsbad, CA, USA) and fetal bovine serum from Wisent Inc. (Montreal, QC, Canada). 3-[4,5-Dimethylthiazol-2-y1]2,5-diphenyltetrazolium bromide (MTT) and dimethyl sulfoxide were obtained from Sigma-Aldrich Co. Giemsa staining solution and 4,6-diamidino-2-phenylindole staining solution were purchased from Beyotime Institute of Biotechnology (Nanjing, People's Republic of China). The cytotoxicity assay kit and Annexin V-fluorescein isothiocyanate apoptosis detection kit were obtained from Beyotime Biotechnology Co., Ltd. (Nantong, People's Republic of China). The lymphoblastoid cells were derived from a Burkitt lymphoma and the resistant cell line to doxorubicin, Raji, and Raji/DNR were provided by the Institute of Hematology, Chinese Academy of Medical Sciences. Standard amino acids, including aspartic acid, glutamic acid, $\gamma$-aminobutyric acid, and glycine were purchased from Sigma (Santa Clara, CA, USA). The hematoxylin-eosin kit was from KeyGen Biotech Co. Ltd. (Nanjing, People's Republic of China). The mouse monoclonal antibodies for Bax, Bcl-2, caspase-3, P-gp, and $\beta$-actin were purchased from Santa Cruz Biotechnology Inc. (Santa Cruz, CA, USA). All regents used in this study were of analytical grade. BALB/c mice, BALB/c-nude mice, and Sprague Dawley rats were purchased from the Shanghai National Center for Laboratory Animals (Shanghai, People's Republic of China) in equal numbers of males and females. $\mathrm{BALB} / \mathrm{c}$ mice and BALB/c-nude mice were 6 weeks old and weighed 18-22 g. Sprague Dawley rats were 7 weeks old and weighed 240-250 g. They were maintained in specific pathogen-free facilities. All animal experiments were performed in accordance with the Animal and Ethics Review Committee of the Affiliated Drum Tower Hospital of Nanjing University Medical School (Nanjing, People's Republic of China).

\section{Synthesis and characterization of DNR- Cys-CdTe and DNR-GA-Cys-CdTe NPs}

The DNR.HCl was efficiently absorbed onto Cys-CdTe QDs via an electrostatic interaction. Briefly, $0.1 \mathrm{~mL}$ of DNR $\cdot \mathrm{HCl}(1 \mathrm{mg} / \mathrm{mL})$ and $0.05 \mathrm{~mL}$ of Cys-CdTe QDs $\left(2.55 \times 10^{-5} \mathrm{M} \text {, calculated according to the literature }\right)^{24}$ were added to $0.85 \mathrm{~mL}$ deionized water and stirred for 22 hours at room temperature in the dark. After being centrifugated at $16,000 \mathrm{rpm}$ for 20 minutes to separate the DNR laden Cys-CdTe NPs, the supernatant DNR was analyzed by fluorescence spectrophotometer (F-4600, Hitachi, Tokyo, Japan). The precipitation was washed three times with deionized water to remove the excess unreacted DNR, and the obtained samples were named as DNR-Cys-CdTe NPs. ${ }^{25}$

For the synthesis of DNR-GA-Cys-CdTe NPs, $0.1 \mathrm{~mL}$ of DNR $\cdot \mathrm{HCl}(1 \mathrm{mg} / \mathrm{mL})$ and $0.05 \mathrm{~mL}$ of Cys-CdTe QDs $\left(2.55 \times 10^{-5} \mathrm{M}\right)$ were added to $0.8 \mathrm{~mL}$ of deionized water, and the mixture was stirred for 6 hours. Then, $0.05 \mathrm{~mL}$ of GA ( $1 \mathrm{mg} / \mathrm{mL})$ was added and the mixture was stirred for another 16 hours. The separation procedures were the same as the synthesis of DNR-Cys-CdTe NPs.

To characterize the DNR-Cys-CdTe and DNR-GA-CysCdTe NPs, the ultraviolet-visible absorption spectra were obtained using an ultraviolet-visible spectrophotometer (UV-3600, Shimadzu, Tokyo, Japan). Fourier transform infrared spectra were recorded on a Nicolet iS10 IR spectrometer (Thermo Scientific, Waltham, MA, USA) in the range of 400-4,000 $\mathrm{cm}^{-1}$, using blank potassium bromide pellet as background. The transmission electron microscopy images were taken by a JEM-2100 transmission electron microscope (JEOL, Tokyo, Japan). The hydrodynamic diameters and size distribution of DNR-GA-Cys-CdTe NPs was measured by dynamic light scattering with a Zetasizer NanoZS size analyzer (Nano ZS90, Malvern Instruments, Malvern, UK).

The drug-loading and encapsulation efficiencies were calculated as follows:

$$
\begin{gathered}
\begin{array}{c}
\text { Loading } \\
\text { efficiency }
\end{array}=\frac{\text { Weight of the drug in NPs }}{\text { Weight of the NPs }} \times 100 \% \\
\begin{array}{c}
\text { Encapsulation } \\
\text { efficiency }
\end{array}=\frac{\text { Weight of the drug in NPs }}{\text { Primary weight of the drug }} \times 100 \%
\end{gathered}
$$

\section{In vitro release from DNR-GA-Cys-CdTe NPs}

In this work, the GA was used as a chemosensitizer to enhance the antitumor effect of DNR. Thus, only the release of DNR from DNR-GA-Cys-CdTe NPs was measured at $\mathrm{pH} 6.0$ (typical $\mathrm{pH}$ of the environment around the tumor) 
and $\mathrm{pH} 7.4$ ( $\mathrm{pH}$ of physiological blood), respectively. The DNR-GA-Cys-CdTe NPs $\left(2.55 \times 10^{-5} \mathrm{M}\right)$ were dispersed in $1 \mathrm{~mL}$ of phosphate-buffered saline (PBS) and transferred into a dialysis bag (molecular weight cutoff of 3,500 Da), which was immersed in $5 \mathrm{~mL}$ of PBS of pH 6.0 and 7.4, followed by continuous shaking $(100 \mathrm{rpm})$ at $37^{\circ} \mathrm{C}$. At predetermined time intervals, aliquots $(0.2 \mathrm{~mL})$ were removed from the buffer solution and an equivalent volume of fresh buffer solution was compensated. The amount of DNR released was then determined by fluorescence spectrophotometer. ${ }^{26}$

\section{Cell cultures}

Human embryonic lung fibroblast (HELF), human lymphoma-resistant cell line to DNR, Raji/DNR, and lymphoma Raji cells (Shanghai Institute of Cells, Chinese Academy of Sciences, Shanghai, People's Republic of China) were grown in RPMI 1640 medium supplemented with $10 \%(\mathrm{v} / \mathrm{v})$ heat-inactivated fetal bovine serum, $10 \mathrm{mM}$ 4-[2-hydroxyethyl]-1-piperazineethanesulfonic acid, $100 \mathrm{U} /$ $\mathrm{mL}$ penicillin, and $100 \mu \mathrm{g} / \mathrm{mL}$ streptomycin in $5 \% \mathrm{CO}_{2}$ and $95 \%$ air at $37^{\circ} \mathrm{C}$ in a humidified incubator. To maintain the drug resistance, Raji/DNR was incubated in a culture containing $1 \mu \mathrm{g} / \mathrm{mL}$ DNR.HCl at least 1 week before use.

\section{Cytotoxicity studies of Cys-CdTe QDs and GA for HELF or Raji/DNR cells in vitro}

MTT assay was used to detect the cytotoxicity and the range of safe concentration of Cys-CdTe QDs and GA. Five-minute sonication of Cys-CdTe QDs were performed before the following experiments. Different concentrations of GA $(0.25,0.5,1$, or $2 \mu \mathrm{g} / \mathrm{mL})$ or Cys-CdTe QDs $(2.5,5,10$, or $20 \mathrm{nmol}$ ) were added to HELF or Raji/DNR cells. Then, these cells were seeded in 96-well plates containing $100 \mu \mathrm{L}$ of $2 \times 10^{5}$ cells suspension and each concentration was set in three wells. In addition, cells added with saline were set as control. After 4 hours of incubation, the adherence appeared. Then, $100 \mu \mathrm{L}$ solution of Cys-CdTe QDs or GA were added to the suspension and incubated at $37^{\circ} \mathrm{C}$ for 24 hours. After that, $20 \mu \mathrm{L}, 5 \mathrm{mg} / \mathrm{mL}$ MTT solution was added to the suspension and cultured for another 4 hours before centrifugation at 1,000 rpm for 10 minutes. At last, $150 \mu \mathrm{L}$ of dimethyl sulfoxide was added to each well and the plates were shaken with a micro-oscillator to dissolve the crystals. An optical density of $540 \mathrm{~nm}$ was measured on a microplate reader (Multiskan MK3, Thermo Scientific, Boston, MA, USA), and the relative growth rate was calculated. Every experiment was repeated at least three times.

\section{Intracellular accumulation of DNR}

Intracellular accumulation of DNR was directly detected by flow cytometry (FCM). Briefly, the Raji/DNR cells were treated as formerly described (described in 'Cell cultures'). After being incubated with $100 \mu \mathrm{L}$ DNR $(2 \mu \mathrm{g} / \mathrm{mL})$, GA+DNR (GA:DNR =1:4 w/w), DNR-Cys-CdTe NPs, or DNR-GA-Cys-CdTe NPs (the concentration of DNR was $2 \mu \mathrm{g} / \mathrm{mL}$ ) for 48 hours, the cells were washed three times with PBS and then suspended in $500 \mu \mathrm{L}$ of PBS. Each sample was measured by FCM(BD FACSAria [TM II]; Becton Dickinson, Franklin Lakes, NJ, USA) at excitation/emission wavelengths of $488 / 575 \mathrm{~nm}$. The relative fluorescence intensity of DNR was computed as $\mathrm{FI}_{\text {treated }}$ group/FI control $_{\text {group. The cells treated }}$ with RPMI 1640 alone were used as control groups.

\section{Cytotoxicity of different agents against Raji and Raji/DNR cells}

After being cultured without drug for 2 weeks, the cells in the log phase were picked in culture flasks and collected by centrifugation in nutrient fluid. The obtained cells were then stained with trypan blue and living cells (more than 98\%) were selected. According to the experiment, the cells were grouped as follows: A) the control group (saline group); B) DNR group; C) DNR-Cys-CdTe NPs group; D) DNR+GA group; and E) DNR-GA-Cys-CdTe NPs group. Every experiment was repeated at least three times.

The cells in log phase were cultured for 4 hours before $100 \mu \mathrm{L}$ DNR $(0.25-8.00 \mu \mathrm{g} / \mathrm{mL}$ for Raji cells and $0.25-50.0 \mu \mathrm{g} / \mathrm{mL}$ for Raji/DNR cells), GA+DNR (GA:DNR =1:4 w/w), DNR-Cys-CdTe NPs, or DNR-GACys-CdTe NPs of different concentrations were added to ensure the same dose of DNR. After incubation for 48 hours, to these cells were added $20 \mu \mathrm{L}, 5 \mathrm{mg} / \mathrm{mL}$ MTT and they were cultured for another 4 hours. Optical density of $540 \mathrm{~nm}$ (A540) was measured after the addition of dimethyl sulfoxide. The half maximal inhibitory concentration $\left(\mathrm{IC}_{50}\right)$ was calculated according to the rate of growth inhibition.

\section{Imaging of Raji/DNR cells by confocal laser scanning microscopy}

Raji/DNR cells $\left(5 \times 10^{4} / \mathrm{mL}\right)$ were cultured in $5 \% \mathrm{CO}_{2}$ at $37^{\circ} \mathrm{C}$ with DNR, Cys-CdTe QDs, or DNR-GA-Cys-CdTe NPs for 12 hours before being gathered through centrifugation at $1,000 \mathrm{rpm}$. After the medium was discarded, the cells were washed at least three times with PBS to remove the unbound drugs. Then, the obtained cell cultures were drop casted on a clean glass slide immediately, followed by the observation with a confocal laser scanning microscope (Fluo View 
FV1000, Olympus, Tokyo, Japan). Excitation of the DNR and Cys-CdTe QDs was performed with lasers at 488 and $350 \mathrm{~nm}$, and emission spectra were collected using a wavelength range of 570-630 nm and 450-500 nm. All the optical measurements were implemented at room temperature $\left(25^{\circ} \mathrm{C} \pm 2^{\circ} \mathrm{C}\right)$.

\section{Apoptosis of Raji/DNR cells}

Raji/DNR cells were cultured with DNR $(2 \mu \mathrm{g} / \mathrm{mL})$, GA+DNR (GA:DNR =1:4 w/w), or DNR-Cys-CdTe or DNR-GA-Cys-CdTe NPs of different concentrations to ensure the same dose of DNR. Cells in different groups were labelled with fluorescein isothiocyanate-conjugated Annexin $\mathrm{V}$ and propidium iodide by using the Annexin V-fluorescein isothiocyanate apoptosis detection kit in accordance with the manufacturer's instructions.

\section{Cell morphological research}

Raji/DNR cells were incubated in RPMI 1640 medium containing DNR, DNR+GA, DNR-Cys-CdTe or DNR-GACys-CdTe NPs (the same concentrations as Apoptosis of Raji/ DNR cells) at $37^{\circ} \mathrm{C}$ for 24 hours. Then, cells were washed three times in PBS and fixed with methanol for 15 minutes. After that, cells were stained with fluorochrome dye 4,6diamidino-2-phenylindole, and subsequently observed under a fluorescence microscope (Imager A1 AX10, Carl Zeiss, Jena, Germany) with an excitation wavelength of $340 \mathrm{~nm}$.

\section{Nude mouse model of MDR lymphoma xenograft}

Thirty nude mice were injected subcutaneously with $0.2 \mathrm{~mL}$ of a DNR-resistant Raji cell suspension $\left(1 \times 10^{7}\right.$ cells $)$. When the tumor size reached $80-150 \mathrm{~mm}^{3}$, the mice were randomly allocated to five groups: group a (saline as negative control), group b (DNR), group c (DNR+GA), group d (DNR-Cys-CdTe NPs), and group e (DNR-GA-Cys-CdTe NPs). Each mouse was intravenously administered seven doses of $0.2 \mathrm{~mL}$ of the treatment agent on days $0,2,4,6,8,10$, and 12 (one dose per day). Based on the drug-loading efficiency, the DNR concentration was $1.2 \mathrm{mg} / \mathrm{kg}$ in each group except the control.

\section{Measurement of tumor size and inhibition rate}

The tumor length (a) and width (b) were measured every day using a caliper and the tumor volume was calculated as follows: $V=1 / 2 \times a \times b^{2}$. Relative tumor volume (RTV) was calculated as follows: $\mathrm{RTV}=\mathrm{Vx} / \mathrm{V} 1$, where $\mathrm{V} 1$ represents the volume on the first day of treatment and $\mathrm{Vx}$ represents the volume on day $\mathrm{X}$. The inhibition rate, reflecting the antitumor effect, was calculated as follows: inhibition rate $=(1-$ mean RTV of the experimental group/mean RTV of the control group) $\times 100 \%$.

\section{Histopathological examination}

After the mice were sacrificed, tumor tissues and organs, including the liver, kidneys, spleen, heart, and lung were isolated from the mice and fixed and embedded. Tissue sections were prepared and stained with hematoxylin and eosin. Histopathological changes were observed by optical microscopy.

\section{Western blotting assay}

The total proteins that would be used for western blotting measurement, were extracted from the tumor tissues of each animal group. Briefly, proteins were size fractionated by sodium dodecyl sulfate polyacrylamide gel electrophoresis and then transferred to a polyvinylidene difluoride membrane. Blotting was performed using monoclonal antibodies (Bax, Bcl-2, caspase-3, P-gp), and 5\% skimmed milk was used as a blocking agent. $\beta$-Actin was used to normalize the data.

\section{Results \\ Synthesis and characterization of DNR-GA-Cys-CdTe NPs}

DNR and GA can be loaded onto the Cys-CdTe QDs to create the DNR-GA-Cys-CdTe NPs due to the electrostatic interaction. ${ }^{27}$ The ultraviolet-vis absorption spectra of DNR, GA, Cys-CdTe QDs, and DNR-GA-Cys-CdTe NPs were measured and are shown in Figure 2A. Except the absorption of Cys-CdTe QDs at $460 \mathrm{~nm}$, two characteristic absorption peaks for DNR (480 nm) and GA (360 nm) were clearly observed in the spectrum of DNR-GA-Cys-CdTe NPs, indicating that the DNR and GA were successfully absorbed by the CysCdTe QDs. In addition, the formation of DNR-GA-Cys-CdTe NPs was confirmed by Fourier transform infrared spectroscopy (Figure 2B). The absorption of DNR on the surface of Cys-CdTe QDs was substantiated by the presence of the characteristic bands of DNR at 1,716 and 1,284 $\mathrm{cm}^{-1}$, which corresponded to the $\mathrm{C}=\mathrm{O}$ stretching vibration and the $\mathrm{O}-\mathrm{H}$ bending vibration in the spectrum of DNR-GA-Cys-CdTe NPs. The characteristic band of GA at $1,680 \mathrm{~cm}^{-1}$ was also observed in the spectrum of DNR-GA-Cys-CdTe NPs and could be assigned to the $\mathrm{C}=\mathrm{C}$ stretching vibration, further supporting the loading of GA. The above results show strong indication that DNR and GA coloaded DNR-GA-Cys-CdTe NPs have been obtained. The size, morphology, and size distribution of these DNR-GA-Cys-CdTe NPs were shown in Figure 2C and D. The DNR-GA-Cys-CdTe NPs exhibited very good 

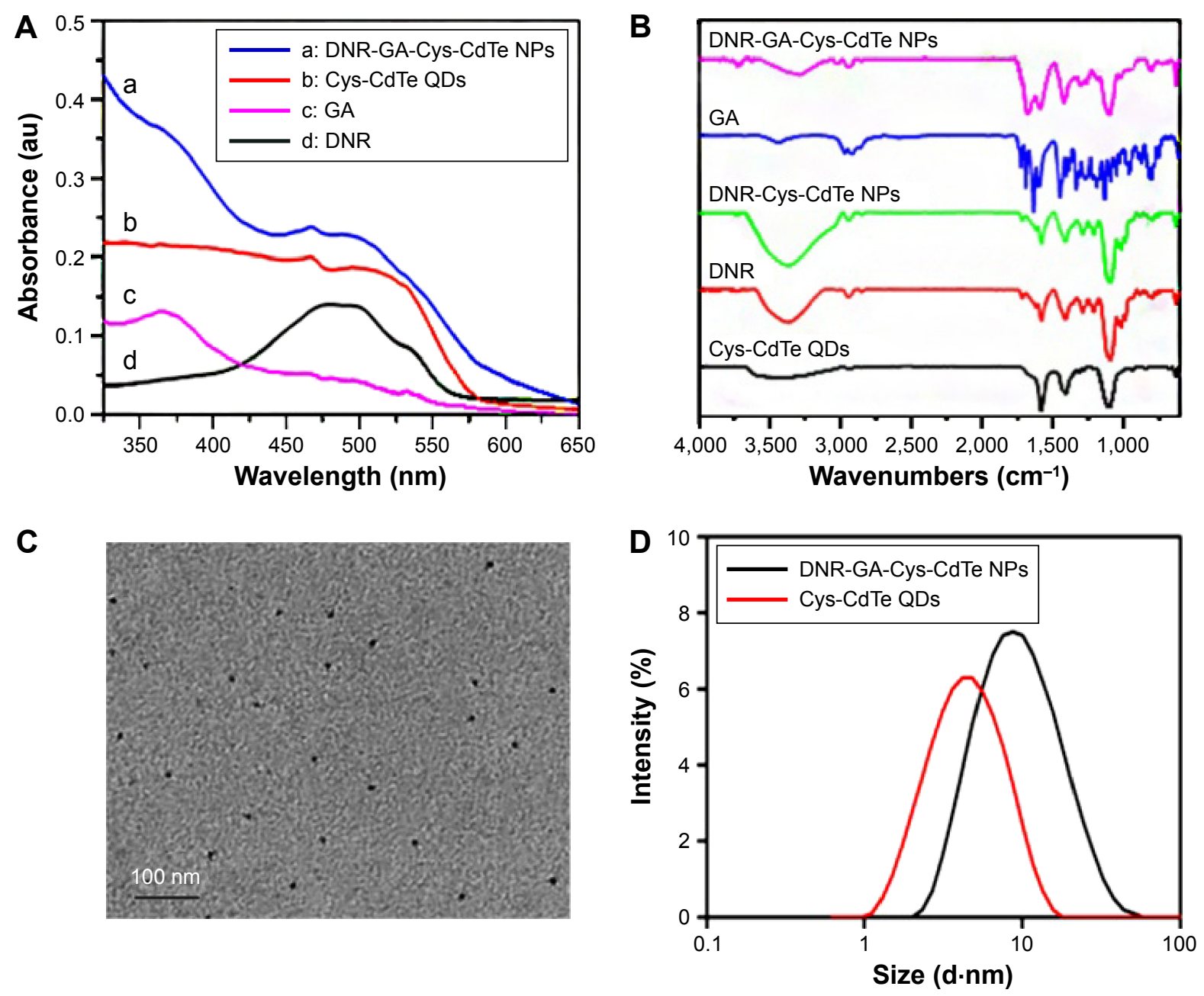

Figure 2 Characterization of DNR-GA-Cys-CdTe NPs.

Notes: (A) Ultraviolet-vis spectra of DNR, GA, Cys-CdTe QDs, and DNR-GA-Cys-CdTe NPs; (B) Fourier transform infrared spectra of DNR-GA-Cys-CdTe NPs, GA, DNR-Cys-CdTe NPs, DNR, and Cys-CdTe QDs; (C) TEM images of DNR-GA-Cys-CdTe NPs; (D) DLS analysis of DNR-GA-Cys-CdTe NPs.

Abbreviations: Cys, cysteamine; CdTe, cadmium-tellurium; DLS, dynamic light scattering; DNR, daunorubicin; EPR, enhanced permeability and retention; GA, gambogic acid; NPs, nanoparticles; QDs, quantum dots; TEM, transmission electron microscopy.

dispersibility in water. Although, some of the NPs aggregated when in dried state, the transmission electron microscopy image of DNR-GA-Cys-CdTe NPs showed spherical morphology with a size around $6 \mathrm{~nm}$ (Figure 2C). The average hydrodynamic size of Cys-CdTe QDs obtained from the dynamic light scattering is $3.21 \mathrm{~nm}$, and the average hydrodynamic size of DNR-GA-Cys-CdTe NPs is $7.2 \mathrm{~nm}$ (Figure 2D). The zeta potential demonstrates that after attachment of drug molecules to the surface of the Cys-CdTe quantum dots, the relevant zeta potential value changed from $-20.4 \pm 4.1$ to $-10.3 \pm 2.3 \mathrm{mV}$.

\section{Encapsulation efficiency and in vitro drug release behavior of DNR from DNR-GA- Cys-CdTe NPs}

The high surface-volume ratio of Cys-CdTe QDs makes it easy to load them with a great quantity of DNR. After the encapsulation of DNR, the encapsulation efficiency and DNR release ability were measured (Table 1). The encapsulation efficiencies of DNR are $92.45 \% \pm 0.85 \%$ (DNR-Cys-CdTe NPs) and $84.47 \% \pm 1.35 \%$ (DNR-GA-Cys-CdTe NPs),

Table I Encapsulation efficiency and loading efficiency of DNR and GA on DNR-Cys-CdTe NPs or DNR-GA-Cys-CdTe NPs

\begin{tabular}{llllll}
\hline & \multicolumn{2}{l}{ DNR } & & GA & \\
\cline { 2 - 3 } & Encapsulation efficiency (\%) & Loading efficiency (\%) & & Encapsulation efficiency (\%) & Loading efficiency (\%) \\
\hline DNR-Cys-CdTe NPs & $92.45 \pm 0.85$ & $48.04 \pm 0.44$ & - & - \\
DNR-GA-Cys-CdTe NPs & $84.47 \pm 1.35$ & $40.04 \pm 0.64$ & $26.49 \pm 0.92$ & $12.56 \pm 0.44$ \\
\hline
\end{tabular}

Abbreviations: Cys, cysteamine; CdTe, cadmium-tellurium; DNR, daunorubicin; GA, gambogic acid; NPs, nanoparticles. 
confirming that Cys-CdTe QDs can be used as efficient anticancer drug carriers. Because of the presence of GA, the encapsulation efficiencies of DNR slightly decreased in the DNR-GA-Cys-CdTe NPs.

The DNR release from the DNR-GA-Cys-CdTe NPs was performed in the medium with different $\mathrm{pH}$ values (Figure 3). The release of DNR depended on the $\mathrm{pH}$ of the media, as well as the time. The release rate of DNR at pH 7.4 was comparatively slow, and only $30 \%$ of the DNR was released from the DNR-GA-Cys-CdTe NPs within 24 hours. But at $\mathrm{pH}$ 6.0, the release of DNR was more rapid, and approximately $90 \%$ of DNR was released into the medium within 24 hours, indicating that the release of DNR from DNR-GA-Cys-CdTe NPs was pH-triggered. This result is quite interesting since the physiological condition of blood is neutral with a $\mathrm{pH}$ of 7.4. Consequently, most of the DNR will remain stable on the surface of the DNR-GA-Cys-CdTe NPs when they are injected into the plasma, which largely reduces the side effects of DNR on the normal cells/tissues during the blood circulation. Due to the enhanced permeability and retention effect, these long-circulation NPs can be passively targeted to the tumor tissue with acidic microenvironment, resulting in a faster drug release. Therefore, an efficient DNR release and accumulation in the target tumor tissues can be obtained. Moreover, GA can effectively bind to transferrin receptor, suggesting that GA may be able to target cancer cells in cancer therapy. In this study, the presence of GA offered DNR-GA-Cys-CdTe NPs the additional ability to bind to cancer cells, which could accelerate the cellular uptake of these NPs. After being taken up by the tumor cells via an

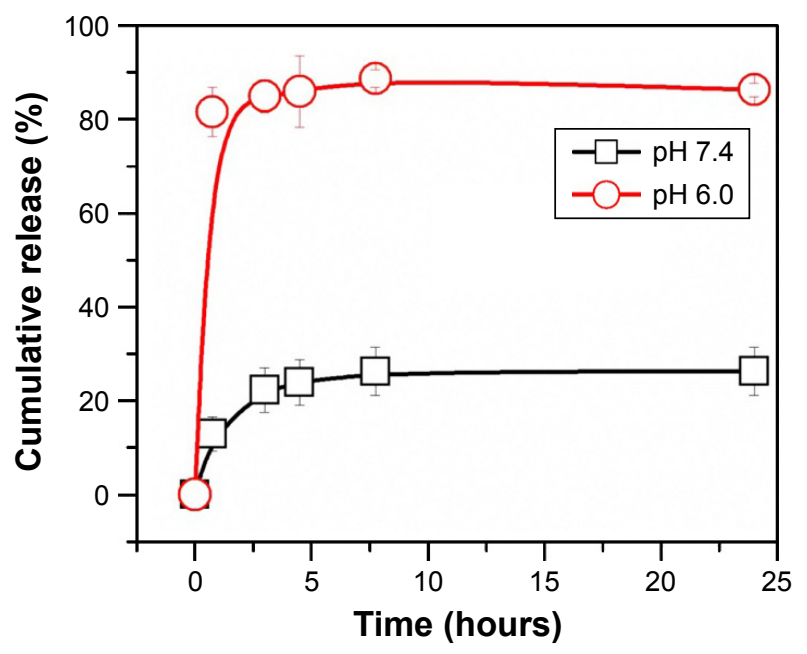

Figure 3 DNR release behavior from DNR-GA-Cys-CdTe NPs at $\mathrm{pH} 7.4$ and $\mathrm{pH} 6.0$.

Abbreviations: Cys, cysteamine; CdTe, cadmium-tellurium; DNR, daunorubicin; GA, gambogic acid; NPs, nanoparticles. endocytosis process, because of the low $\mathrm{pH}$ value inside the endocytic compartments ( $\mathrm{pH}$ from 4.5 to 6.5 ), a much faster DNR release was obtained. Therefore, a sufficiently high concentration of DNR could be obtained inside the tumor cells within a short period of time, which also helped to minimize MDR.

\section{Cytotoxicity of Cys-CdTe QDs and GA for HELF and Raji/DNR cells}

Prior to in vivo application, the cytotoxicity of Cys-CdTe QDs and GA was tested by MTT assay. Although, QDs were thought not to be biocompatible and had cytotoxicity at high concentrations, the Cys-CdTe QDs showed an inhibition $<10 \%$ against HELFs in the test concentration ranging from 2.5 to $10 \mathrm{nmol}$. Increasing the concentration to $20 \mathrm{nmol}$ resulted in a lower cell viability of $<80 \%$, which indicated that Cys-CdTe QDs could be safely used at a concentration $<20$ nmol. On the other hand, in this study, GA was used as a sensitizer to improve the sensitivity of DNR and strengthen the therapeutic efficacy against the lymphoma. However, GA also showed significant anticancer activity in vitro and in vivo with high dose, which would interfere with the antitumor effect of DNR. So in order to avoid the inhibitory effect of GA on the tumor cells, the maximum concentration of GA, which could maintain its sensitive effect and avert the antitumor ability, was tested. Figure 4 shows the cytotoxicity of GA against HELF and Raji/DNR cells with different concentrations. The GA showed a dose-dependent cytotoxicity. The treatment of HELF and Raji/DNR cells with $2.0 \mu \mathrm{g} / \mathrm{mL}$ of GA did not bring significant inhibition effect on the cells $(P>0.05)$. Thus, the highest concentrations of GA and Cys-CdTe QDs were chosen to be $<0.5 \mu \mathrm{g} / \mathrm{mL}$ and $20 \mathrm{nmol}$, respectively, which would impose little side effect on the proliferation of the HELFs.

\section{Intracellular accumulation of DNR}

The cellular uptake of different NPs samples was measured by FCM. Figure 5 shows the concentration of DNR in the Raji/DNR cells after these cells were incubated with different samples for 48 hours. Compared with the control group whose fluorescence intensity was defined as 1 , the relative fluorescence intensities are $9.34 \pm 1.73,16.75 \pm 1.47$, $14.66 \pm 2.98$, and $26.37 \pm 2.28$ for DNR, DNR+GA, DNR-CysCdTe, and DNR-GA-Cys-CdTe NPs groups, respectively. These results indicated that by coloading GA and DNR onto the Cys-CdTe QDs, the highest concentration of DNR was detected in the Raji/DNR cells treated with DNR-GACys-CdTe NPs, indicating that the presence of GA could strengthen the cellular uptake of DNR-GA-Cys-CdTe NPs. 

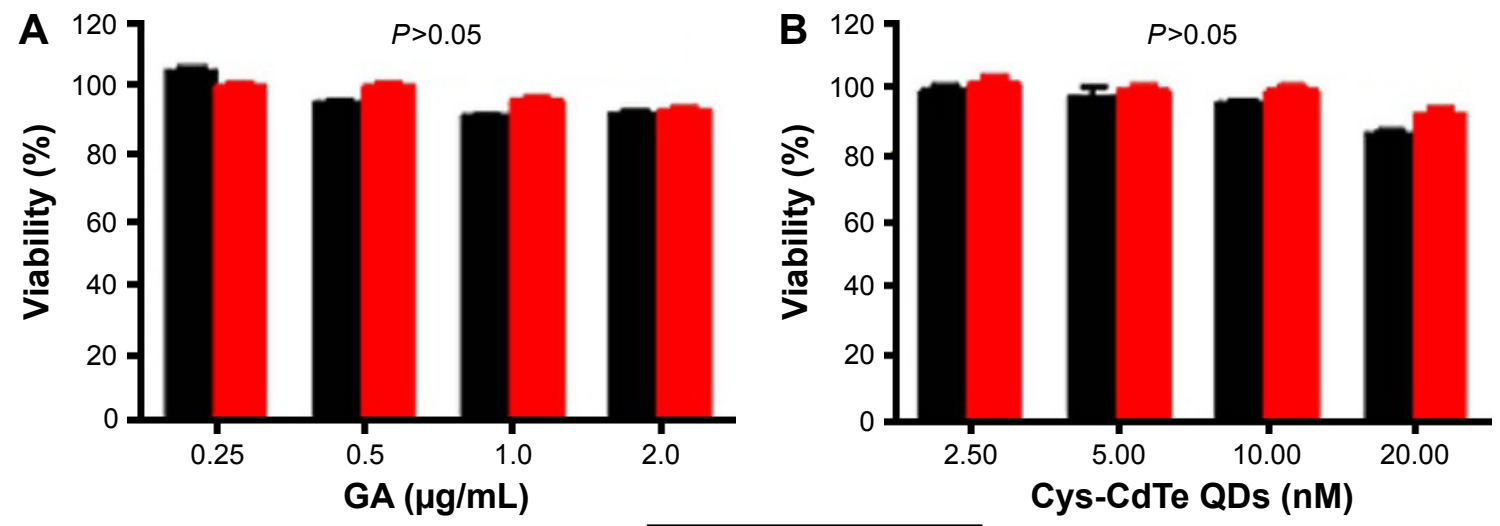

HELF Raji/DNR

Figure 4 The cytotoxicity of GA (A) or Cys-CdTe QDs (B) for HELF and Raji/DNR cells with different concentration in 24 hours ( $P>0.05$ ).

Abbreviations: Cys, cysteamine; CdTe, cadmium-tellurium; DNR, daunorubicin; GA, gambogic acid; HELF, human embryonic lung fibroblast; QDs, quantum dots.
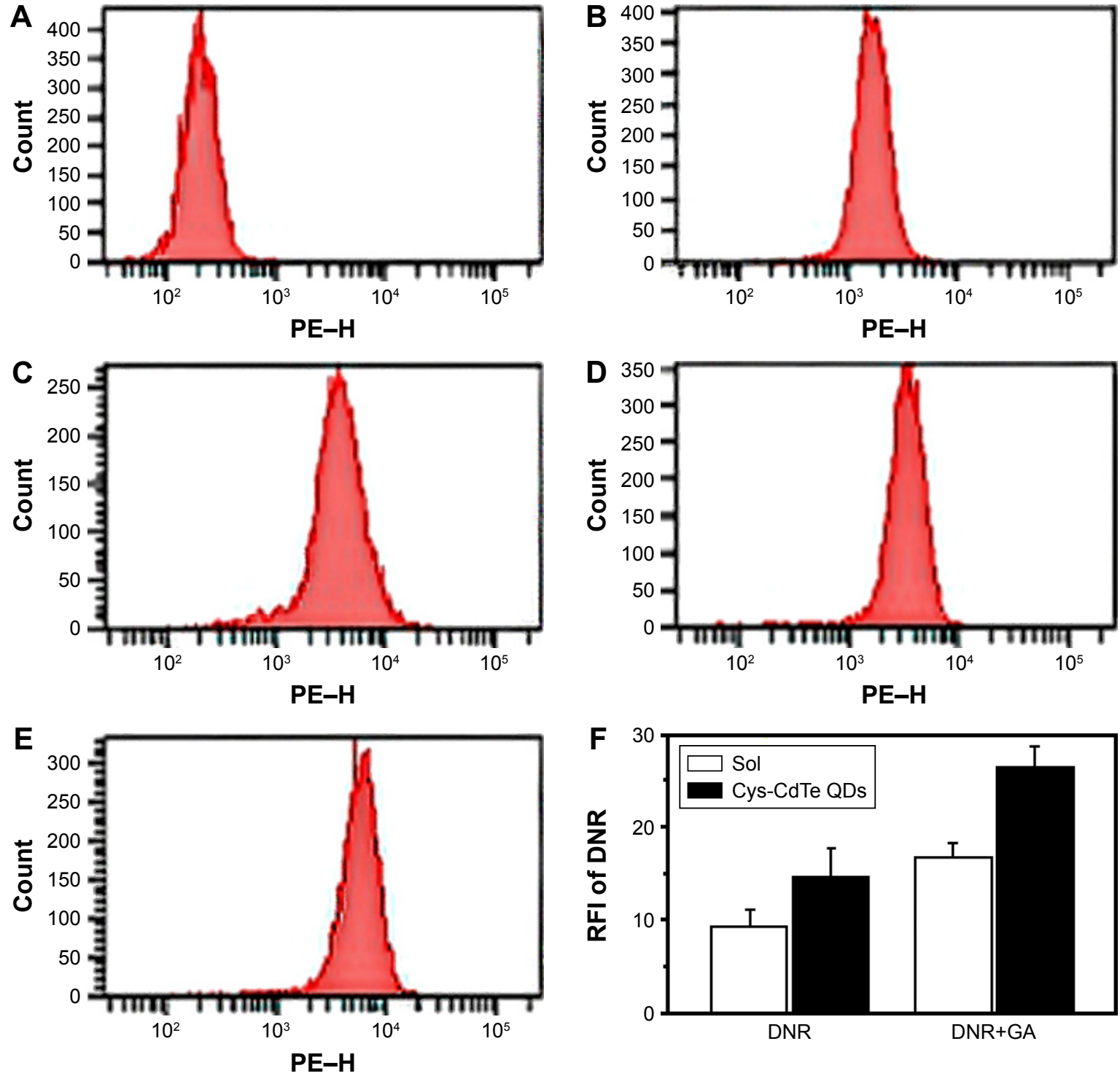

Figure 5 Intracellular accumulation of DNR with different treatment evaluated by $\mathrm{FCM}(P<0.05)$.

Notes: (A) Control; (B) DNR; (C) DNR+GA; (D) DNR-Cys-CdTe NPs; (E) DNR-GA-Cys-CdTe NPs; (F) relative fluorescence intensity (RFI) of DNR in Raji/DNR cells; sol: DNR or DNR+GA alone.

Abbreviations: Cys, cysteamine; CdTe, cadmium-tellurium; DNR, daunorubicin; FCM, flow cytometry; GA, gambogic acid; NPs, nanoparticles. 


\section{Cytotoxicity of different agents for Raji and Raji/DNR cells}

The cytotoxicity of DNR, DNR-Cys-CdTe NPs, DNR+GA, and DNR-GA-Cys-CdTe NPs against lymphoma cells was measured, and results are shown in Figure 6A. For the Raji cells treated with different samples, cytotoxicity was dose-dependent and almost the same in the tested concentration. But for the drug-resistant Raji/DNR cells, DNR had the lowest cytotoxicity while DNR-GA-Cys-CdTe NPs exhibited the highest cytotoxicity (Figure 6B). The $\mathrm{IC}_{50}$ of DNR, DNR-Cys-CdTe NPs, DNR+GA, and DNRGA-Cys-CdTe NPs in Raji/DNR cells was 20.128, 7.762, 3.043 , and $1.255 \mu \mathrm{g} / \mathrm{mL}$, respectively, which confirmed that the cytotoxicity of DNR against the drug-resistant human lymphoma cells was notably enhanced with the presence of Cys-CdTe QDs and GA.

\section{Fluorescence imaging and cellular labeling}

Compared with traditional organic dyes, QDs have unique advantages as fluorescence probes. We further explored the cellular labeling and imaging functions of DNR-GACys-CdTe NPs. Raji/DNR cells were incubated with DNR, Cys-CdTe QDs, and DNR-GA-Cys-CdTe NPs and then examined by confocal microscope. As shown in Figure 7, after incubation with Cys-CdTe QDs, the weak blue fluorescence in the lymphoma cells was easily caught by confocal laser scanning microscopy, indicating successful penetration of Cys-CdTe QDs into Raji/DNR cells. Moreover, when treated with DNR-GA-Cys-CdTe NPs, the lymphoma cells could be clearly observed through fluorescence of these NPs (Figure 7D). Since GA molecules had no fluorescence and the fluorescent background of the cell had been removed, the intracellular fluorescence was only produced by DNR and Cys-CdTe QDs. ${ }^{28}$ In addition, the presence of multiple purple spots in the overlap image from DNR channel (red) and Cys-CdTe QDs channel (blue) clearly indicates that DNR is loaded on Cys-CdTe QDs and the appearance of DNR and GA did not influence the fluorescence of Cys-CdTe QDs. All the results suggested that it may be possible to use DNR-GA-Cys-CdTe NPs in cancer therapy as well as nanoprobes for real-time labeling and imaging of cancer cells.

\section{Apoptosis of Raji/DNR cells}

FCM was used to quantitatively investigate the apoptosis of Raji/DNR cells (Figure 8). After being incubated for 48 hours, the total apoptosis of Raji/DNR cells was $2.94 \% \pm 0.17 \%$ in the control group. Additionally, the apoptosis of cells in the DNR group increased to $8.69 \% \pm 0.36 \%$ but showed no significant difference with the blank group, suggesting that DNR cannot induce apoptosis of Raji/ DNR cells. When the cells were cultured with DNR+GA and DNR-Cys-CdTe NPs, the apoptosis increased to $28.80 \% \pm 0.45 \%$ and $40.13 \% \pm 2.30 \%$, respectively. Furthermore, when Raji/ DNR cells were treated by DNR-GA-Cys-CdTe NPs, the rate of apoptosis increased to $50.55 \% \pm 1.84 \%$, which showed a significant change compared with the control group. These results proved that GA could increase the ability of DNR to induce apoptosis of Raji/DNR cells. Moreover, when DNR, with or without GA, was loaded on the surface of Cys-CdTe QDs, the apoptosis of cells remarkably increased, suggesting that Cys-CdTe QDs could be used as a drug delivery system.
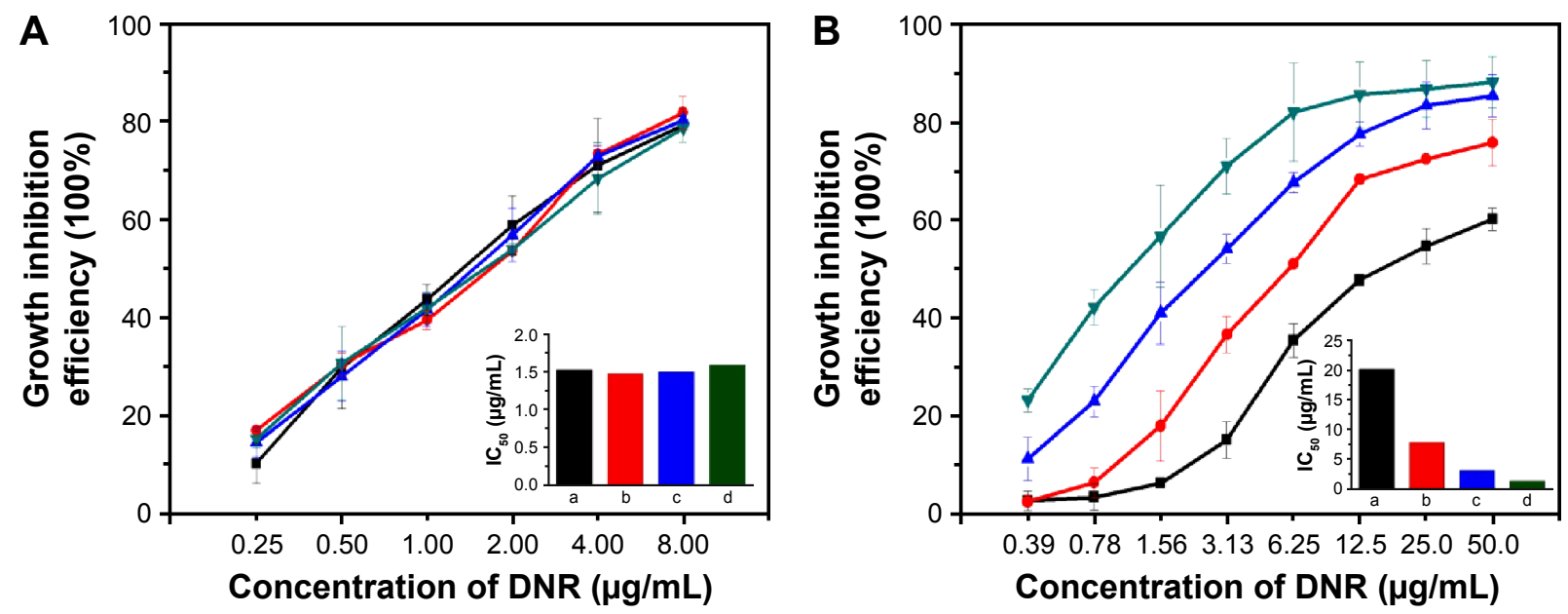

$\rightarrow$ DNR $\rightarrow$ DNR-Cys-CdTe NPs $\leftarrow$ DNR+GA $\multimap$ DNR-GA-Cys-CdTe NPs

Figure 6 Inhibition profiles of DNR, DNR-Cys-CdTe NPs, DNR+GA, and DNR-GA-Cys-CdTe NPs for (A) Raji and (B) Raji/DNR cells. Notes: IC f $_{50}$ of DNR, DNR-Cys-CdTe NPs, DNR+GA, and DNR-GA-Cys-CdTe NPs for (A) Raji $(P>0.05)$ and $(\mathbf{B})$ Raji/DNR cells $(P<0.05)$. Abbreviations: Cys, cysteamine; CdTe, cadmium-tellurium; DNR, daunorubicin; GA, gambogic acid; NPs, nanoparticles. 


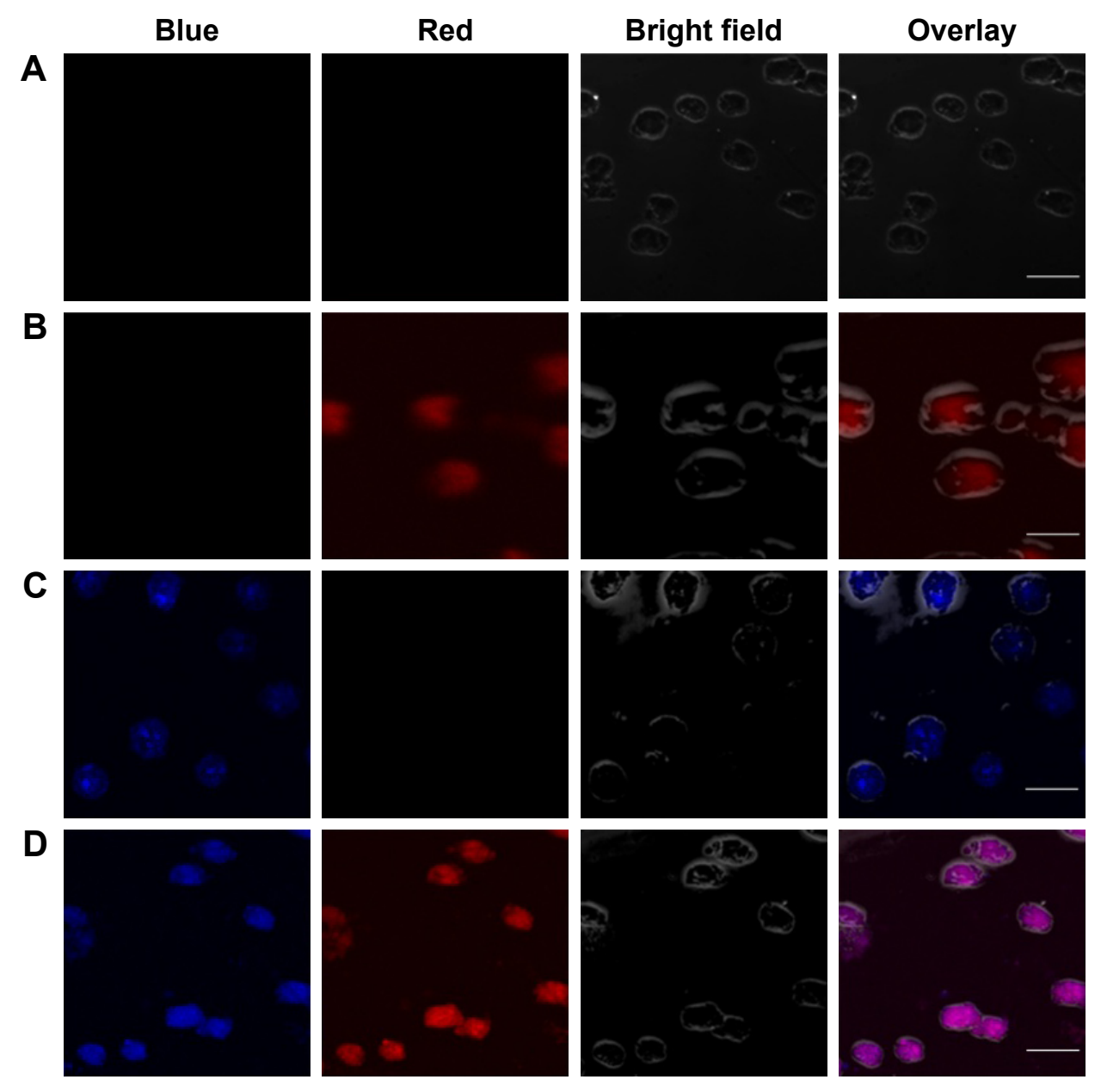

Figure 7 Confocal fluorescence microscopy images of Raji/DNR cells QDs.

Notes: (A) Control; (B) DNR; (C) Cys-CdTe QDs; (D) DNR-GA-Cys-CdTe NPs. Scale bar $=10 \mu \mathrm{m}$.

Abbreviations: Cys, cysteamine; CdTe, cadmium-tellurium; DNR, daunorubicin; GA, gambogic acid; NPs, nanoparticles; QDs, quantum dots.

\section{Cancer cell apoptosis induced by DNR-GA-Cys-CdTe NPs}

The morphological changes of these drug-resistant Raji/DNR cells were further explored to study cancer cell apoptosis using fluorescent microscopy. As shown in Figure 9A, Raji/DNR cells in the control group emitted blue fluorescence, which was homogeneously dispersed inside the nucleus without remarkable cellular morphology variation, indicating no occurrence of apoptosis. After these cells were treated with DNR (Figure 9B or DNR+GA, the fluorescence intensity of 4,6-diamidino-2-phenylindole inside nucleus became stronger, but the increase of apoptosis was not significant. However, after incubation with DNR-Cys-CdTe NPs (Figure 9D) or DNR-GA-Cys-CdTe NPs (Figure 9E) for 24 hours, these Raji/DNR cells emitted much stronger/brighter fluorescence inside the nucleus, and the typical phenomena of apoptosis, including nucleolus pyknosis, chromatin condensation, and nuclear fragmentation were clearly observed (indicated by red arrows). Also, the cells ruptured and were unable to maintain their normal shape (Figure 9E). These observations attested the synergistic anticancer effect due to the codelivery of GA and DNR by DNR-GA-Cys-CdTe NPs. Higher amount of DNR delivered into the lymphoma cells by such a system can result in higher cytotoxicity, which consequently inhibits cellular proliferation.

\section{Evaluation of tumor inhibition in a nude mouse model of MDR lymphoma xenograft}

Over the course of 14 days posttreatment, the nude mice were in good condition without obvious adverse reactions. As shown in Figure 10, the tumor volume decreased gradually in the DNR-Cys-CdTe- and the DNR-GA-Cys-CdTe-treated groups, and the RTVs in these two groups were smaller than those of the other three groups $(P<0.05)$, especially in the DNR-GA-Cys-CdTe group $(P<0.01)$ (Figure 10). 
A

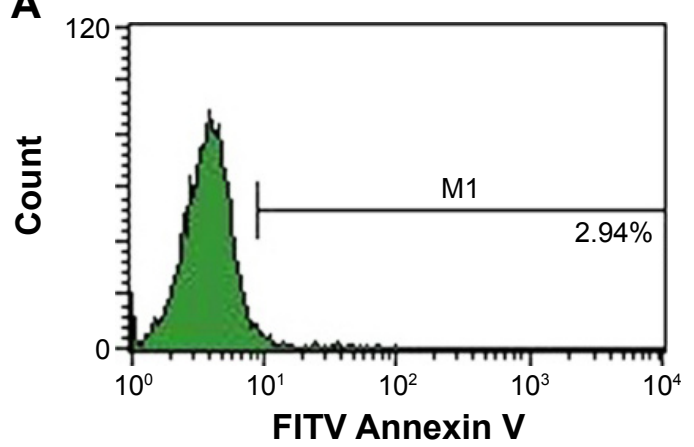

C

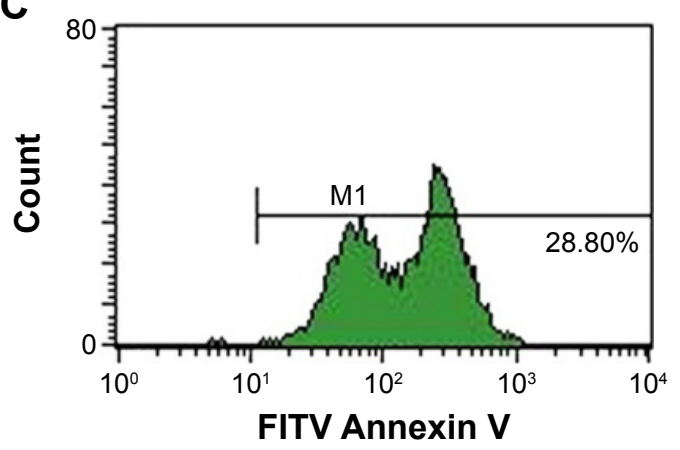

B

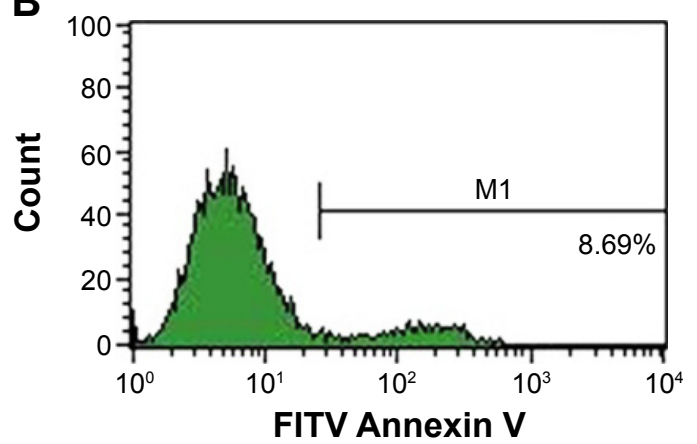

D

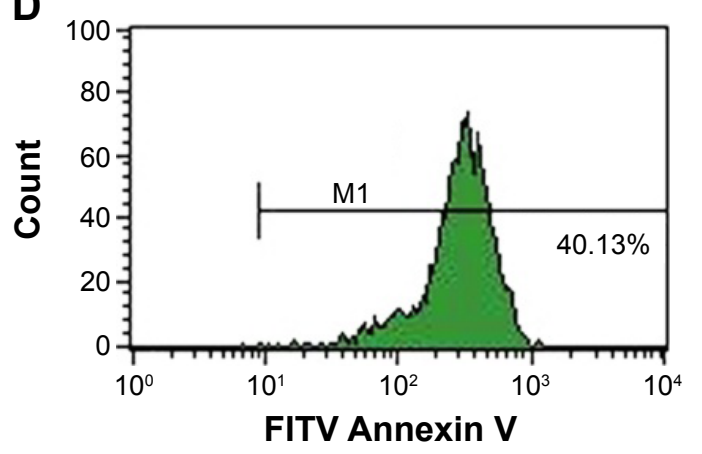

E

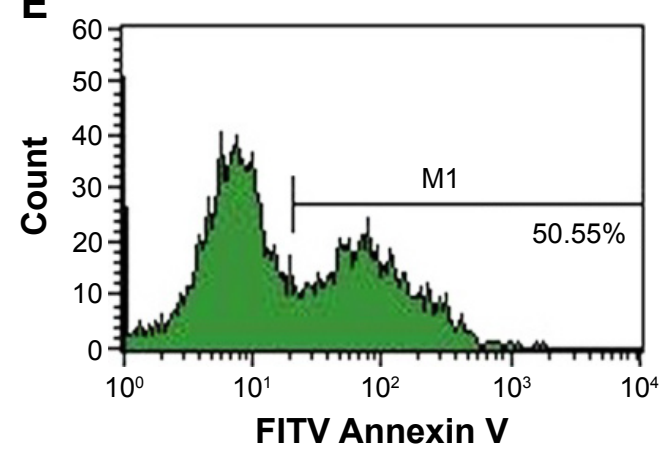

Figure 8 The apoptosis of Raji/DNR cells with different treatment evaluated by FCM.

Notes: (A) Control; (B) DNR; (C) DNR+GA; (D) DNR-Cys-CdTe NPs; (E) DNR-GA-Cys-CdTe NPs.

Abbreviations: Cys, cysteamine; CdTe, cadmium-tellurium; DNR, daunorubicin; FCM, flow cytometry; FITC, fluorescein isothiocyanate; GA, gambogic acid; NPs, nanoparticles.

At day 6 posttreatment, the tumor inhibition rates in the DNR-GA-Cys-CdTe, DNR-Cys-CdTe, DNR+GA, and DNR groups were $87.29 \% \pm 3.77 \%, 58.49 \% \pm 3.65 \%$, $22.02 \% \pm 5.22 \%$, and $9.33 \% \pm 0.58 \%$ (Figure 10C), respectively, suggesting that DNR-GA-Cys-CdTe NPs had the highest tumor inhibition ability. Tumor tissues were isolated after the mice were sacrificed. DNR-GA-Cys-CdTe NPs-treated group had the smallest tumor among all the samples (Figure 10A), indicating that DNR-GA-Cys-CdTe NPs could suppress tumor growth and enhance the antitumor activity of DNR.

\section{Histopathological examination}

In the saline and DNR groups, the isolated tumor tissues presented a ruddy appearance with abundant blood supply, and tumor cells appeared in a disordered arrangement, with different sizes, heterogeneous cell types, numerous mitoses, and large hyperchromatic nuclei upon histopathological examination. In contrast, sheet necrosis and few neovessels were observed in the DNR-Cys-CdTe NPs- and DNR-GACys-CdTe NPs-treated groups (Figure 10B).

\section{Expressions of Bax, Bcl-2, caspase-3, and P-gp in tumor tissues}

Western blotting was performed to detect the protein expression of Bax, Bcl-2, caspase-3, and P-gp in tumor tissues (Figure 11). There was no significant difference for all detected proteins between the saline and DNR groups $(P>0.05)$, but all protein expression levels in the DNR+GA 

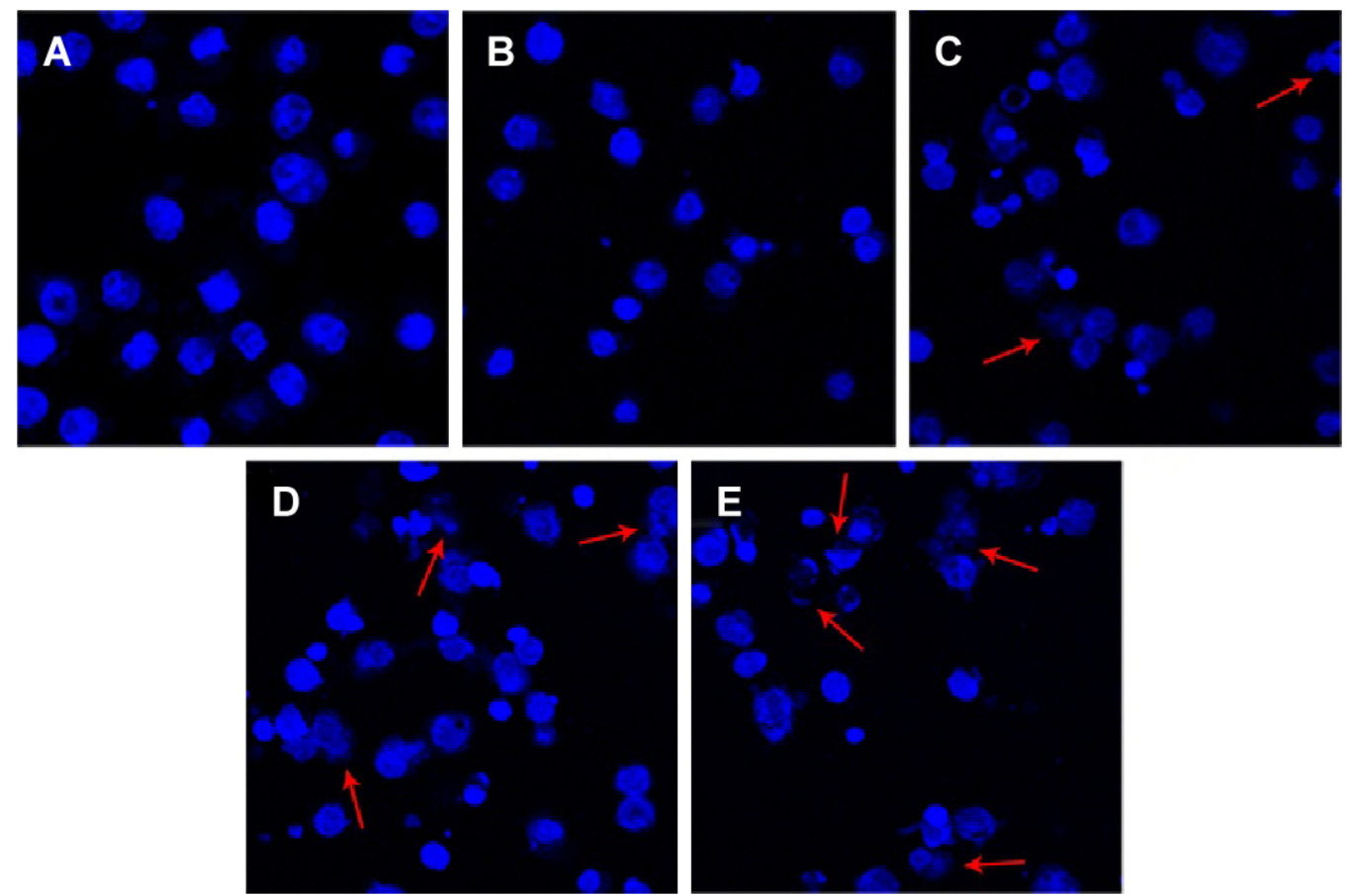

Figure 9 Fluorescence microscope images of (A) control, (B) DNR, (C) DNR+GA, (D) DNR-Cys-CdTe, and (E) DNR-GA-Cys-CdTe NPs. (400x). Note: The typical phenomena of apoptosis were indicated by red arrows.

Abbreviations: Cys, cysteamine; CdTe, cadmium-tellurium; DNR, daunorubicin; GA, gambogic acid; NPs, nanoparticles.

A

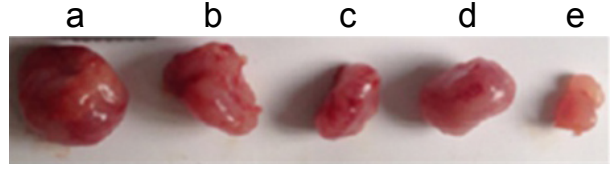

B

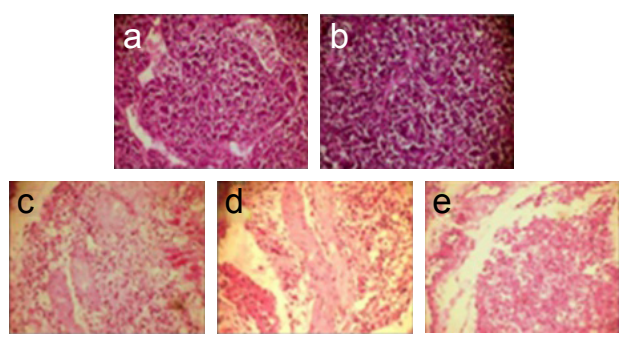

C

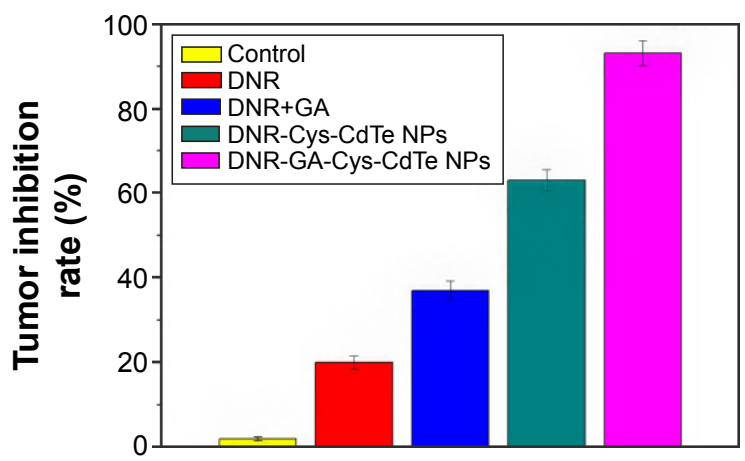

D

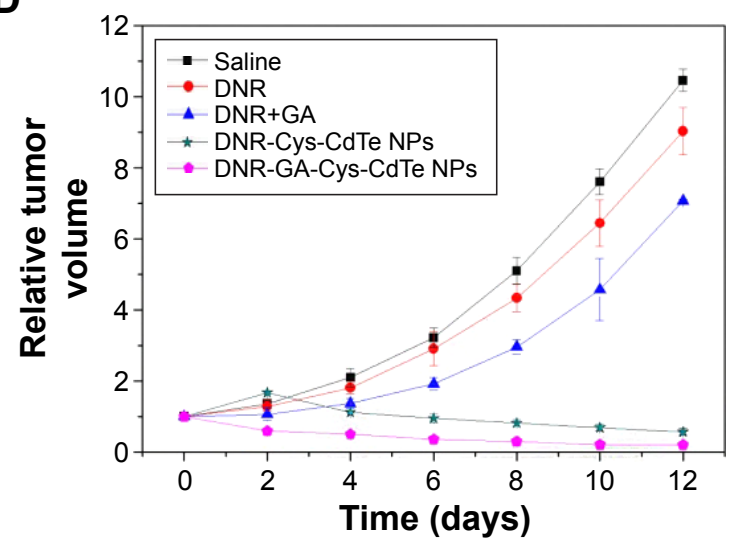

Figure 10 Tumor inhibition in a nude mouse model of DNR-resistant Raji xenograft after treatment.

Notes: (A) Isolated tumor bodies. (B) Histopathological examination of tumor tissues in the mouse model at day 12 posttreatment (hematoxylin and eosin staining, $\times 40$ ). (C) Tumor inhibition rates of different groups; (D) Relative tumor volumes of different treatments: Saline; DNR; DNR+GA; DNR-Cys-CdTe NPs; DNR-GA-Cys-CdTe NPs. Abbreviations: Cys, cysteamine; CdTe, cadmium-tellurium; DNR, daunorubicin; GA, gambogic acid; NPs, nanoparticles. 


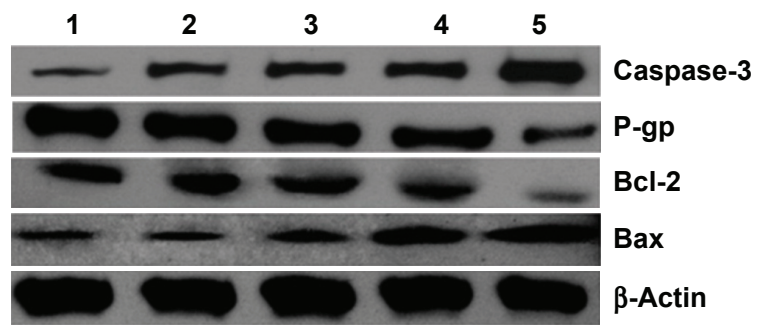

Figure I I The expressions of apoptosis-related proteins (Bax, Bcl-2, and caspase-3) and P-gP after treatment. I, saline; 2, DNR; 3, DNR+GA; 4, DNR-Cys-CdTe NPs; 5, DNR-GA-Cys-CdTe NPs. The results were normalized by $\beta$-actin expression. Abbreviations: Cys, cysteamine; CdTe, cadmium-tellurium; DNR, daunorubicin; GA, gambogic acid; NPs, nanoparticles.

group were significantly different from the saline and DNR groups $(P<0.05)$. In the DNR-Cys-CdTe and DNR-GA-CysCdTe groups, Bax and caspase-3 were strongly expressed, while the expression of Bcl-2 and P-gp decreased significantly $(P<0.05)$, which resulted in an elevated ratio of Bax/ Bcl-2 $(P<0.01)$ compared with those of the other groups.

\section{Discussion}

Although systemic chemotherapy is still the main therapy option for NHL, drug-resistant cells usually lead to the therapy failure. P-gp plays an important role in developing chemotherapeutic resistance because it can pump anticancer drugs out of the cellular membrane. ${ }^{29} \mathrm{~A}$ lot of chemotherapeutic agents (anthracyclines or paclitaxel) have been testified to induce drug resistance via overexpressing $\mathrm{P}$-gp, which is the main mechanism of MDR..$^{30} \mathrm{~A}$ variety of compounds (MDR reversal agents) are applied to interfere with P-gp function. Such agents may improve the efficacy of conventional therapy when they are used in combination with anticancer drugs. As a chemotherapy sensitization agent, GA can inhibit the activation of nuclear factor- $\mathrm{\kappa B}$ and increase the accumulation of anticancer drugs inside cells through downregulating the level of P-gp. Besides, nanotechnology also holds promising potential to minimize MDR and decrease the side effects in the cancer therapy. ${ }^{31}$ In this work, DNR-GA-Cys-CdTe NPs were fabricated with the aim to minimize P-gp-mediated MDR in Raji/DNR cells. Compared with coadministration of a MDR reversal agent and an anticancer drug, coloading of GA and DNR onto nanoparticles can deliver these drugs locally and simultaneously, thus maximizing the therapeutic effect. ${ }^{32}$

The in vitro drug release of DNR from DNR-GACys-CdTe NPs was pH sensitive. Considering the acidic microenvironment around in the tumor tissue and inside the subcellular organelle (endosome, lysosome), most of the loaded DNR would be released in the tumor or inside the tumor cells, while imposing less side effects of DNR on the normal tissue. The highest relative fluorescence intensity of DNR inside the cells treated with DNR-GA-Cys-CdTe NPs also proved that presence of GA significantly increased the intracellular concentration of DNR, resulting in the highest growth inhibition efficiency. The antitumor effect on nude mice also showed that DNR-GA-Cys-CdTe NPs had the best tumor inhibition. The results of Western blot confirmed the lowest level of P-gp in the Raji/DNR cells treated by DNRGA-Cys-CdTe NPs, clearly indicating the ability of GA to reverse the MDR effect of Raji/DNR cells through inhibiting the expression of P-gp on the cell membrane.

\section{Conclusion}

In summary, DNR-GA-Cys-CdTe NPs were prepared and used as multifunctional deliver platform to deliver DNR and trace cancer cells in cancer therapy. These DNR-GACys-CdTe NPs demonstrated good aqueous dispersion and efficient drug-loading ability. They could enable rapid drug release in an acidic environment and induce excellent antitumor effect in vitro. It is evident that coloading of DNR and GA with Cys-CdTe QDs can significantly increase DNR accumulation inside the drug-resistant Raji/DNR cells, and thus increase the cytotoxicity of DNR. Above all, these DNR-GA-Cys-CdTe NPs are able to minimize the MDR of Raji/DNR cells and efficiently inhibit the proliferation of cancer cells. This formulation will be a promising strategy for minimizing MDR and open a new avenue toward systemic chemotherapy for NHL.

\section{Acknowledgments}

This work was supported by the National Natural Science Foundation of People's Republic of China (Nos 81400162, 21474047), the Natural Science Foundation of Jiangsu Province (BK20140100), and the Technique Development Foundation of Nan Jing (Outstanding Youth Foundation, JQX15004).

\section{Disclosure}

The authors report no conflicts of interest in this work.

\section{References}

1. Gelfand JM, Shin DB, Neimann AL, Wang XM, Margolis DJ, Troxel AB The risk of lymphoma in patients with psoriasis. J Invest Dermatol. 2006; 126(10):2194-2201.

2. Siegel RL, Miller KD, Jemal A. Cancer statistics, 2015. CA Cancer J Clin 2015;62(1):5-29.

3. Bhatt VR, Vose JM. Hematopoietic stem cell transplantation for non-Hodgkin lymphoma. Henatol Oncol Clin North Am. 2014;28(6): 1073-1095. 
4. Boland MP, Foster SJ, O'Neill LAJ. Daunorubicin activates NF kappa B and induces kappa B-dependent gene expression in HL-60 promyelocytic and Jurkat T lymphoma cells. J Biol Chem. 1997;272(20):12952-12960.

5. Tulpule A, Rarick MU, Kolitz J, et al. Liposomal daunorubicin in the treatment of relapsed or refractory non-Hodgkin's lymphoma. Ann Oncol. 2001;12(4):457-462.

6. Lothstein L, Israel M, Sweatman TW. Anthracycline drug targeting: cytoplasmic versus nuclear - a fork in the road. Drug Resist Updat. 2001;4(3):169-177.

7. Szakács G, Paterson JK, Ludwig JA, Booth-Genthe C, Gottesman MM. Targeting multidrug resistance in cancer. Nat Rev Drug Discov. 2006; 5(3):219-234.

8. Gottesman MM, Fojo T, Bates SE. Multidrug resistance in cancer: role of ATP dependent transporters. Nat Rev Cancer. 2002;2(1):48-58.

9. Loo TW, Clarke DM. Recent progress in understanding the mechanism of P-glycoprotein-mediated drug efflux. J Member Biol. 2005;206(3): 173-185.

10. Klopman G, Shi LM, Ramu A. Quantitative structure-activity relationship of multidrug resistance reversal agents. Mol Pharmacol. 1997; 52(2):323-334.

11. Fox E, Bates SE. Tariquidar (XR9576): a P-glycoprotein drug efflux pump inhibitor. Expert Rev of Anticancer Ther. 2007;7(4):447-459.

12. Li XL, Lu XW, Xu HE, et al. Paclitaxel/tetrandrine coloaded nanoparticles effectively promote the apoptosis of gastric cancer cells based on "oxidation therapy". Mol Pharm. 2012;9(2):222-229.

13. Sun MJ, Wang J, Lu Q, et al. Novel synthesizing method of pHdependent doxorubicin-loaded anti-CD22-labelled drug delivery nanosystem. Drug Des Devel Ther. 2015;9(2):5123-5133.

14. Schroeder U, Sommerfeld P, Ulrich S, Sabel BA. Nanoparticle technology for delivery of drugs across the blood-brain barrier. J Pharm Sci. 1998; 87(11):1305-1307.

15. Xu PP, Li JY, Shi LX, Selke M, Chen BA, Wang XM. Synergetic effect of functional cadmium-tellurium quantum dots conjugated with gambogic acid for HepG2 cell-labeling and proliferation inhibition. Int J Nanomedicine. 2013;8:3729-3736.

16. Li JY, Wu CY, Xu PP, et al. Multifunctional effects of Cys-CdTe QDs conjugated with gambogic acid for cancer cell tracing and inhibition. RSC Adv. 2013;3(18):6518-6525.

17. Wang LH, Yang JY, Yang SN, et al. Suppression of NF-KB signaling and P-glycoprotein function by gambogic acid synergistically potentiates adriamycin-induced apoptosis in lung cancer. Curr Cancer Drug Targets. 2014;14(1):91-103.

18. Wang TT, Wei J, Qian XP, Ding YT, Yu LX, Liu BR. Gambogic acid, a potent inhibitor of surviving, reverses docetaxel resistance in gastric cancer cells. Cancer Lett. 2008;262(2):214-222.
19. Yong KT, Law WC, Roy I, et al. Aqueous phase synthesis of CdTe quantum dots for biophotonics. J Biophotonics. 2011;4(1-2):9-20.

20. Li ZS, Xu W, Wang YT, et al. Quantum dots loaded nanogels for low cytotoxicity, $\mathrm{pH}$-sensitive florescence, cell imaging and drug delivery. Carbohydr Polym. 2015;121:477-485.

21. Li JY, Shi LX, Shao YX, et al. The cellular labeling and $\mathrm{pH}$-sensitive responsive-drug release of celastrol in cancer cells based on Cys-CdTe QDs. Sci China Chem. 2014;57(6):833-841.

22. Kasibhatla S, Jessen KA, Maliartchouk S, et al. A role for transferrin receptor in triggering apoptosis when targeted with gambogic acid. Proc Natl Acad Sci U S A. 2005;102(34):12095-12100.

23. Gaponik N, Talapin DV, Rogach AL, et al. Thiol-capping of CdTe nanocrystals: an alternative to organometallic synthetic routes. $J$ Phys Chem B. 2002;106(29):7177-7185.

24. Yu WW, Qu LH, Guo WZ, Peng X. Experimental determination of the extinction coefficient of CdTe, CdSe, and CdS nanocrystals. Chem Mater. 2003;15(14):2854-2860.

25. Wang YZ, Zhang XN, Xu ZL, Huang HL, Li YP, Wang JY. A pHsensitive theranostics system based on doxorubicin conjugated with the comb-shaped polymer coating of quantum dots. J Mater Sci. 2014; 49(21):7539-7546.

26. Shi JJ, Liu Y, Wang L, et al. A tumoral acidic $\mathrm{pH}$-responsive drug delivery system based on a novel photosensitizer (fullerene) for in vitro and in vivo chemo-photodynamic therapy. Acta Biomater. 2014;10(3): 1280-1291.

27. Qu LZ, Deng DW, Wang J, Gu YQ. Dox-conjugated high-quality $\mathrm{AgZnInS}$ QDs for reversal for multidrug resistance. J Innov Opt Health Sci. 2014;7(1):1350043.

28. Li JY, Wu CH, Gao F, et al. In vitro study of drug accumulation in cancer cells via specific association with $\mathrm{CdS}$ nanoparticles. Bioorg Med Chem Lett. 2006;16(18):4808-4812.

29. Jin J, Wang FP, Wei HL, Liu GT. Reversal of multidrug resistance of cancer through inhibition of P-glycoprotein by 5-bromotetrandrine. Cancer Chem Pharm. 2005;55(2):179-188

30. Horwitz SB, Cohen D, Rao S, Ringle I, Shen HJ, Yang CP. Taxol: mechanisms of action and resistance. J Natl Cancer Inst. 1993;15:55-61.

31. Gao ZB, Zhang LN, Sun YJ. Nanotechnology applied to minimize tumor drug resistance. J Control Release. 2012;162(1):45-55.

32. Iyer AK, Khaled G, Fang J, Maeda H. Exploiting the enhanced permeability and retention effect for tumor targeting. Drug Discov Today. 2006; 11(17-18):812-818.
International Journal of Nanomedicine

\section{Publish your work in this journal}

The International Journal of Nanomedicine is an international, peerreviewed journal focusing on the application of nanotechnology in diagnostics, therapeutics, and drug delivery systems throughout the biomedical field. This journal is indexed on PubMed Central, MedLine, CAS, SciSearch ${ }^{\circledR}$, Current Contents ${ }^{\circledR} /$ Clinical Medicine,

\section{Dovepress}

Journal Citation Reports/Science Edition, EMBase, Scopus and the Elsevier Bibliographic databases. The manuscript management system is completely online and includes a very quick and fair peer-review system, which is all easy to use. Visit http://www.dovepress.com/ testimonials.php to read real quotes from published authors. 